\title{
Water film area and dust removal efficiency of string grilles: a theoretical analysis
}

\author{
Hua Guo ${ }^{1,2} \cdot$ Haiqiao Wang ${ }^{1}$ Shiqiang Chen ${ }^{3,4} \cdot$ Zhirong $\mathrm{Wu}^{1}$
}

Received: 1 May 2020/Revised: 19 August 2020/Accepted: 29 April 2021 / Published online: 3 June 2021

(C) The Author(s) 2021

\begin{abstract}
Based on multiphase flow theory and capillary mechanics, the dimensionless bond number expression of the influence of string grille wire spacing on droplet spreading is derived. Taking a liquid film formed by spreading droplets based on Kelvin correlation, the Young-Laplace equation, and the Hagen-Poiseuille law, an equation for calculating the thickness and height of the liquid film is established with temperature, relative humidity and molar volume of liquid phase as independent variables. According to the theory of string grille filtration and dust removal, a dust removal efficiency calculation model covering the wet string grille wire group is constructed based on the liquid film thickness, height, wire diameter, water film area, and vortex shedding frequency. Finally, a theoretical analysis of the influence of water film area on the efficiency of wet string grille dust removal is carried out based on the spray pressure and the ratio of string grille wire distance to wire diameter. It is found that the effect of spray pressure on water film area and dust removal efficiency is more significant than the string grille wire distance diameter ratio. Moreover, the optimized combination of wet string grille wire distance diameter ratio 0.84 , wind speed $3 \mathrm{~m} / \mathrm{s}$ and spray pressure $0.8 \mathrm{MPa}$ is found, which could provide an important reference for engineering applications.
\end{abstract}

Keywords String grille $\cdot$ Wetting mechanism $\cdot$ Capillary action $\cdot$ Water film area $\cdot$ Dust removal efficiency

\section{Abbreviations}

a Height of the single layer string grille $(\mathrm{mm})$

$b \quad$ Width of the single layer string grille $(\mathrm{mm})$

Shiqiang Chen

shiqiangchen@hnust.edu.cn

Hua Guo

ghaq1989@163.com

1 School of Resource, Environment and Safety Engineering, Hunan University of Science and Technology, Xiangtan 411201, Hunan, China

2 School of Civil and Environmental Engineering, Hunan University of Science and Engineering, Yongzhou 425199, Hunan, China

3 Hunan Provincial Engineering Techniques Research Centre of Mine Ventilation and Dedusting Equipment, Hunan University of Science and Technology, Xiangtan 411201, Hunan, China

4 Work Safety Key Lab On Prevention and Control of Gas and Roof Disasters for Southern Coal Mines, Hunan University of Science and Technology, Xiangtan 411201, Hunan, China
A Filter area $\left(\mathrm{m}^{2}\right)$

$A_{\mathrm{w}} \quad$ Effective area function of the water film $\left(\mathrm{m}^{2}\right)$

$B_{\mathrm{O}} \quad$ Bond number (dimensionless)

$C \quad$ Dust concentration entering the unit (\%)

$C_{0} \quad$ Dust concentration in front of the dust flow through the plate $\left(\mathrm{kg} / \mathrm{m}^{3}\right)$

$C_{1} \quad$ Dust concentration after dust flow through the plate $\left(\mathrm{kg} / \mathrm{m}^{3}\right)$

$C_{\mathrm{u}} \quad$ Cunningham correction coefficient (dimensionless)

$d_{0} \quad$ Distance between the two adjacent string grille wires (mm)

$d_{\mathrm{x}} \quad$ Unit filter (dimensionless)

$d_{\mathrm{f}} \quad$ String grille wire diameter $(\mathrm{mm})$

$d_{\mathrm{p}} \quad$ Dust particle size $(\mathrm{mm})$

$F_{\mathrm{g}} \quad$ Water mist gravity $(\mathrm{Pa})$

$F_{\mathrm{m}} \quad$ Negative pressure difference of the total water film (Pa)

$F_{v} \quad$ Adjacent string grille wire viscosity force $(\mathrm{Pa})$

$g \quad$ Gravitational acceleration $(\mathrm{N} / \mathrm{kg})$

$h^{1} \quad$ Height of the water film when it does not reach equilibrium $(\mathrm{mm})$ 
$h \quad$ Rising water film height in the string grille wire clearance (mm)

$H \quad$ Hamaker constant $(\mathrm{J})$

$L \quad$ Water film thickness (mm)

$L_{\mathrm{s}} \quad$ Total length of the double string grille wire diameter (mm)

$p \quad$ Water mist pressure $(\mathrm{MPa})$

$P_{0} \quad$ Separation pressure in the water film $(\mathrm{Pa})$

$p_{\mathrm{g}} \quad$ Saturated vapor pressure of the water mist $(\mathrm{Pa})$

$p_{1} \quad$ Saturated vapor pressure of the water $(\mathrm{Pa})$

$Q \quad$ Dust gas flow $\left(\mathrm{m}^{3} / \mathrm{s}\right)$

$r_{0} \quad$ Radial coordinates $(\mathrm{m})$

$r \quad$ Average curvature radius $(\mathrm{mm})$

$r_{1} \quad$ Curvature radius of the water film side curved surface perpendicular ( $\mathrm{mm})$

$r_{2}$ Curvature radius of the water film side curved surface parallel (mm)

$r_{\mathrm{k}} \quad$ Kelvin radius $(\mathrm{m})$

$R \quad$ Low interception settlement (dimensionless)

$R_{0} \quad$ Universal gas constant $\left(\mathrm{J} \mathrm{K}^{-1} \mathrm{~mol}^{-1}\right)$

$R e$ Reynolds number (dimensionless)

$S_{\mathrm{r}} \quad$ Vortex shedding frequency (dimensionless)

$S_{\mathrm{tk}} \quad$ Stokes number (dimensionless)

$S_{\text {we }}$ Effective saturation of the water on the plate (dimensionless)

$S_{\mathrm{w}} \quad$ Water saturation (dimensionless)

$S_{\mathrm{w} 0} \quad$ Irreducible saturation of water (dimensionless)

$T \quad$ Absolute temperature (K)

$V_{\mathrm{m}} \quad$ Molar volume of the water mist $\left(\mathrm{m}^{3} / \mathrm{mol}\right)$

$z \quad$ Capillary tube length direction coordinate $(\mathrm{m}$

\section{Greek symbols}

$\alpha \quad$ Correction coefficient (dimensionless)

$\alpha_{\mathrm{d}} \quad$ String grille wire distance diameter ratio (dimensionless)

$\beta_{\mathrm{s}} \quad$ Wire-filling rate $(\%)$

$\beta_{\mathrm{ws}} \quad$ Filling rates of the wire and water $(\%)$

$\beta_{\mathrm{w}} \quad$ Filling rate of water on string grille wire (\%)

$\delta \quad$ Surface tension of the water film $(\mathrm{N} / \mathrm{m})$

$\delta_{\mathrm{lv}} \quad$ Liquid gas surface tension $(\mathrm{N} / \mathrm{m})$

$\Delta p \quad$ Negative pressure difference $(\mathrm{Pa})$

$\varepsilon \quad$ Wet string grille voidage $(\%)$

$\varepsilon_{\mathrm{s}} \quad$ String grille voidage $(\%)$

$\eta_{1} \quad$ Filtration efficiency of each string grille wire (\%)

$\eta_{\mathrm{f}} \quad$ Dust capture efficiency of simplified string grille wire $(\%)$

$\eta_{\mathrm{w}} \quad$ Dust removal efficiency of water film (\%)

$\eta_{\mathrm{wf}} \quad$ Dedusting efficiency of the double-layer wet string grille $(\%)$

$\theta \quad$ Angle $\left(^{\circ}\right)$

$\lambda_{\mathrm{c}} \quad$ Capillary length $(\mathrm{mm})$

$\mu \quad$ Viscosity of water mist (Pa S)

$\mu_{\mathrm{g}} \quad$ Viscosity of air ( $\left.\mathrm{Pa} \mathrm{S}\right)$ $v \quad$ Incoming flow velocity $(\mathrm{m} / \mathrm{s})$

$\bar{v} \quad$ Average velocity $(\mathrm{m} / \mathrm{s})$

$\rho \quad$ Water mist density $\left(\mathrm{kg} / \mathrm{m}^{3}\right)$

$\rho_{\mathrm{p}} \quad$ True density of dust particles $\left(\mathrm{kg} / \mathrm{m}^{3}\right)$

$\tau \quad$ Internal friction force on the wall of the string grille wire $(\mathrm{Pa})$

$\varphi \quad$ Dust collected by the string grille wire per unit length $(g)$

\section{Introduction}

In the process of mining, a large amount of dust is produced, which arrives at the airshaft because of the underground air flow and is then discharged (Yao et al. 2020). This leads to atmospheric pollution. Various scholars have developed mine dust removal methods and these can mainly be divided into dry and wet dust removal variants (Reed et al. 2019; Zhang et al. 2020). String grille dust removal technology is favored because it is suitable for large air volumes and high levels of pollution in a limited space. String grille dust removal technology is a type of filtering dust removal technology. The droplet group ejected from the nozzle collides with the surface of the string grille, and the droplets are capillary wetted on the surface of the string grille due to the capillary effect. Under the pneumatic disturbance of the dust laden air flow, a downward flowing water film is formed in the longitudinal fine string grille gap. When the dust laden air passes through the water film, it is captured by the water film and flows downward with the water flow; the fracture is generated and new water film is generated continuously under the action of the continuous spray droplet group. During this process, the dynamics of wet string grille water film dust removal mainly depend on the water film area, which directly affects the dedusting efficiency of the string grille.

In terms of wet dust removal, Zhou et al. (2017, 2020) and $\mathrm{Li}$ et al. (2017) explored spray pressure. The spray dedusting technology was optimized to render it amenable in practical contexts, resulting in a high dust removal efficiency. Further, Wang et al. (2019) and Han et al. (2020) analyzed the relationship between the atomization characteristics of the atomizing nozzle and the dust removal efficiency. Fairs (1958) discovered the existence of a liquid film by studying the formation of a sulfuric acid vinegar fog on four kinds of fibers with different properties. Using a continuous spray of water mist on the fibers, Agranovski and Braddock (1998) designed a method to form a water film after a few minutes. Theoretical and experimental studies show that the water film thickness increases with fiber equivalent diameter and differs depending on the height of the fiber grille. In the case of 
equilibrium saturation, Jakub (2015), Jakub et al. (2016) and Ryan et al. (2013) observed uneven liquid coverage on the fiber and studied the efficiency of a filter-trapping aerosol. Gong (2002) studied the dominant mechanism of water film formation on the fiber grille and pointed out the capillary effect of the fiber spacing. Tan and Jiang (2015) and Jin et al. (2010) theoretically analyzed the dedusting mechanism of water films and assumed that dust particles that are difficult to moisten tend to penetrate into the water film. Li et al. (2011) and Wu et al. (2017) explained dust removal efficiency from the perspective of the fiber grille gap, material, and grille, as well as nozzle spacing according to water films observed in experiments. However, most of these studies analyzed the existence of water films from a macro and indirect perspective and there remains a lack of systematic analysis of the physical mechanism of water film formation and fragmentation processes, and thus the relationship between water film state and dust removal efficiency is poorly understood.

To study the relationship between water film and dust removal efficiency, it is important to understand the interaction between string grille structure and droplets. In this paper, a theoretical analysis is carried out concerning the process of removing a water film from the string grille. Mathematical expressions of the water film area and the dust removal efficiency are established by analyzing the influence rule of the water film area on the spray pressure, the wind speed, and the structural parameters of the string grille. By limiting the wind speed, dust particle size, and water irreducible saturation, the efficiency of dust removal and the water film area are analyzed by changing the spray pressure and the string grille wire distance diameter ratio. It is concluded that both of these variables have a significant effect on the water film area and the efficiency of dust removal, which provides a reference for theoretical and engineering applications of string grille dust removal.

\section{Materials and methods}

\subsection{Water film formation mechanism}

The water mist contacts the two adjacent wires and forms a water film owing to surface adhesion forces and capillary action. The Bond number is used to describe the capillary phenomenon in surface mechanics and is the relative magnitude of static water mist gravity and surface tension (Zhuang et al. 2016):

$B \mathrm{o}=\frac{\rho g \lambda_{\mathrm{c}}^{2}}{\delta_{\mathrm{lv}}}$

where, $\lambda_{\mathrm{c}}$ is capillary length, which is considered here to be half of the string grille wire spacing $d_{0}(\mathrm{~mm}), \rho$ is the water mist density $\left(\mathrm{kg} / \mathrm{m}^{3}\right), g$ is gravitational acceleration $(\mathrm{N} / \mathrm{kg})$, and $\delta_{\mathrm{lv}}$ is the liquid gas surface tension $(\mathrm{N} / \mathrm{m})$. When the Bond number is large, surface tension produced by static water mist under capillary pressure can be ignored. A small Bond number $(<0.1)$ shows that the surface tension strongly affects the hydrostatic gravity.

In the film formation test, the water is $20^{\circ} \mathrm{C}, \delta_{\mathrm{lv}}=7.3 \times$ $10^{-3} \mathrm{~N} / \mathrm{m}, g=9.8 \mathrm{~N} / \mathrm{kg}, \rho=994 \mathrm{~g} / \mathrm{m}^{3}$, combined with reasonable reference values and $B \mathrm{o}=0.0036$ (Probmtein et al. 1992). From Eq. (1), the distance between the string grille wire is calculated from the equations above to be less than $0.34 \mathrm{~mm}$. The water mist generates a capillary phenomenon between the string grille wire.

\subsection{Basic physical parameters of the string grille}

The diameter of the string grille wire is a particularly important physical parameter. The string grille wire is cylindrical in shape and uniformly distributed in the wire grille with equal spacing. To facilitate the formation of a capillary water film on the string grille and improve the dust removal efficiency of the string grille, the distance of the string grille wire must be less than $0.34 \mathrm{~mm}$. The diameter of the string grille wire is represented by $d_{\mathrm{f}}$, and the ratio of string grille wire distance to diameter is expressed in $\alpha_{\mathrm{d}}$. According to analysis of the filtering mechanism of the string grille, the string grille wire is uniformly distributed on the rectangular string grille and parallel to the airflow direction. The percentage of the string grille wire area to the whole string grille area prior to wetting is expressed by $\beta_{\mathrm{s}}$. The wire-filling rate is determined during production. String grille voidage $\varepsilon_{\mathrm{s}}$ is obtained according to $1-\beta_{\mathrm{s}}$.

When the airflow carries water mist through the string grille, the filling rate of the wet string grille wire includes the water mist attached to the string grille wire and the residual water film after the film ruptures. The filling rate of the string grille is therefore composed of the filling rates of the unwetted wire and water:

$\beta_{\mathrm{ws}}=\beta_{\mathrm{s}}+\beta_{\mathrm{w}}$

$\varepsilon=1-\beta_{\mathrm{ws}}$

where, $\beta_{\mathrm{ws}}$ and $\beta_{\mathrm{w}}$ are the filling rates of the wet wire and water, respectively (dimensionless). The liquid holding capacity of the string grille wire is measured and calculated experimentally and $\varepsilon$ represents the wet string grille voidage (dimensionless). 


\subsection{Calculation of water film thickness and height on a string grille}

When the water mist and string grille wire meet conditions of film formation, a liquid film force is produced, also known as a liquid bridge force (Gao and Liu 2010; Tanaka and Iwamoto 2016). Capillary action theory can be used to analyze the liquid film force. The left and right sides of the water film between the string grille wire are connected owing to adhesion. On the other hand, there is surface tension on the upper and lower film surfaces, which forms a concave water film surface in the arc (Fig. 1) and leads to a pressure difference between the gas and body side of the water film (i.e., the pressure outside the water film is greater than that inside). This negative pressure on the film itself has a pulling effect, which is essentially the liquid film force.

\section{(1) Derivation and calculation of water film thickness}

During the tests, water mist is sprayed continuously on the string grille plate with a small gap between the wire, and a water film forms under capillary action. The outer outline of the water film between the wire is a circular arc concave surface owing to the influence of the water film force (Fig. 2). Let $d_{0}$ represent the distance between the two adjacent string grille wires. The average curvature radius of

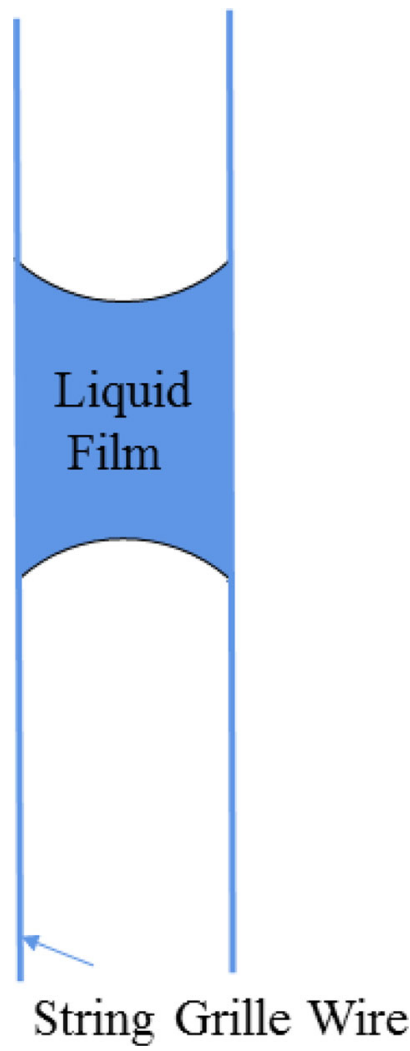

Fig. 1 Concave water film surface

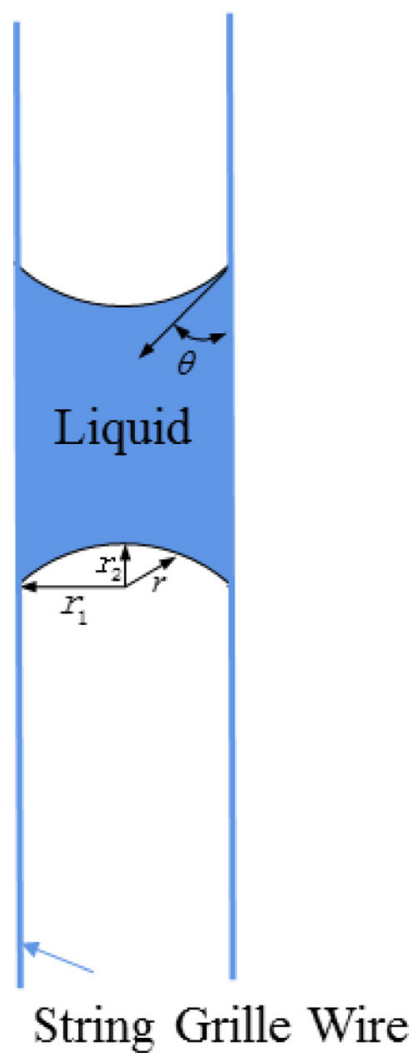

Fig. 2 Radius of the concave liquid surface

the water film side surface is $r, r_{1}$ and $r_{2}$ are the curvature radius of the curved surface perpendicular and parallel to the string grille wire, respectively, and the surface tension of the water film is $\delta$. According to the Young-Laplace equation (Lin et al. 2015), the negative pressure difference of the water film between the string grille wire is as follows:

$\Delta p=\frac{2 \delta}{r}=\delta\left(\frac{1}{r_{1}}+\frac{1}{r_{2}}\right)$

In a humid environment, when the water mist and parallel body of the wettable string grille wire are close to one another, the string grille surface absorbs the water mist molecules to form a water film. According to physical and mechanical knowledge of surfaces and interfaces, the separation pressure $P_{0}$ in the water film is expressed as

$P_{0}=\frac{H}{6 \pi L^{3}}$

where, $H$ is the Hamaker constant $(\mathrm{J})$ and $L$ is the water film thickness (mm).

Under mechanical equilibrium conditions, the negative pressure of the water film between the string grille wire is equal to the separation pressure in the water film. Combined with the relationship between the curvature radius of the water film surface and relative humidity in the 
continuous spray environment, the Kelvin equation (Adamson and Gast 1997)shows

$r_{\mathrm{k}}=\left(\frac{1}{r_{1}}+\frac{1}{r_{2}}\right)^{-1}=\frac{\delta V_{\mathrm{m}} \cos \theta}{R_{0} T \ln \left(p_{\mathrm{g}} / p_{1}\right)}$

where, $r_{\mathrm{k}}$ is the Kelvin radius $(\mathrm{m}), V_{\mathrm{m}}$ is the molar volume of the water mist $\left(\mathrm{m}^{3} / \mathrm{mol}\right), \theta$ is the angle, taken here as $0^{\circ}$, $R_{0}$ is the universal gas constant $\left(\mathrm{J} \cdot \mathrm{K}^{-1} \cdot \mathrm{mol}^{-1}\right), T$ is the absolute temperature $(\mathrm{K}), p_{\mathrm{g}}$ is the saturated vapor pressure of the water mist $(\mathrm{Pa}), p_{1}$ is the saturated vapor pressure of the water $(\mathrm{Pa})$, and $p_{\mathrm{g}} / p_{1}$ is the saturated vapor pressure ratio of water mist to water (dimensionless). Fitting the reference data (Wang 2007), the relationship between the function of saturated vapor pressure ratio and the spray pressure $p(\mathrm{MPa})$ can be obtained as follows:

$\frac{p_{\mathrm{g}}}{p_{\mathrm{l}}}=0.9425 p^{-0.467}$

The negative pressure difference of the water membrane and the separation pressure are a pair of equilibrium forces which can be determined by combining Eqs. (4)-(7):

$\delta \frac{R_{0} T \ln \left(0.9425 p^{-0.467}\right)}{\delta V_{\mathrm{m}}}=\frac{H}{6 \pi L^{3}}$

The thickness of the available water film is

$L=\left[\frac{H}{6 \pi} \cdot \frac{V_{\mathrm{m}}}{R_{0} T \ln \left(0.9425 p^{-0.467}\right)}\right]^{\frac{1}{3}}$

Under continuous spraying at $20{ }^{\circ} \mathrm{C}$, the molar volume of water fog is $V_{m}=1.8 \times 10^{5} \mathrm{~m}^{3} / \mathrm{mol}$, the universal gas constant $R_{O}=8.31 \mathrm{~J} \mathrm{~K}^{-1} \mathrm{~mol}^{-1}$, the absolute temperature $T=293 \mathrm{~K}$, and the Hamack constant of water mist $H=3.72 \times 10^{-20} \mathrm{~J}$.

(2) Derivation and calculation of water film height

Water mist between the wire forms a water film under capillary action and appears as a circular concave liquid surface under magnification. The height of the concave water film is affected by the total capillary force (negative pressure difference of the total water film) $F_{\mathrm{m}}$, adjacent string grille wire viscosity force $F_{v}$, and water mist gravity $F_{\mathrm{g}}$.

According to the Young-Laplace equation (Lin et al. 2015), the capillary force (negative pressure difference of the water film) can be written as follows:

$\Delta p=\frac{2 \delta}{r_{2}}$

As can be seen in Fig. 2, $r_{2}=\frac{r}{\cos \theta}$

If the distance between the string grille wire is $d_{0}=2 r$, the total capillary force is as follows:

$F_{\mathrm{m}}=2 \pi r \delta \cos \theta$

The capillary motion of the water mist between string grille wires is unsteady and satisfies the Navier-Stokes equation. To simplify the calculation, the flow of water mist between the string grille wires is regarded as steady, the radial and circumferential direction components of the flow velocity are set to 0 , and the flow is symmetrically distributed. The Navier-Stokes equation (Zhang et al. 2018) can therefore be expressed by the special Hagen-Poiseuille equation (Chen and Yao 2017). The viscous force between string grille wires can be obtained by the internal friction law of a Newtonian viscous fluid and the Hagen-Poiseuille equation. The constitutive equations of Newtonian viscous fluid are as follows:

$\tau=\left.\mu \frac{\mathrm{d} v}{\mathrm{~d} r_{0}}\right|_{r_{0}=r}$

where, $\tau$ is the internal friction force on the wall of the string grille wire $(\mathrm{Pa}), \mu$ is the viscosity of water mist ( $\mathrm{Pa}$ $\mathrm{S}), v$ is the incoming flow velocity $(\mathrm{m} / \mathrm{s})$, and $r_{0}$ is the radial coordinates $(\mathrm{m})$. The above formulations show that the viscous internal friction force of the water mist on the surface of the wire is linearly related to the velocity gradient, and the distribution law of the flow rate under wind action is similar to that in a circular tube. The HagenPoiseuille equation (Chen and Yao 2017) can be used to express

$\frac{1}{r} \frac{\mathrm{d}}{\mathrm{d} r_{0}}\left(r_{0} \frac{\mathrm{d} v}{\mathrm{~d} r_{0}}\right)=\frac{1}{\mu} \frac{\partial p}{\partial z}$

where, $p$ is the spray pressure $(\mathrm{MPa})$ and $z$ is the capillary tube length direction coordinate $(\mathrm{m})$.

Taking into account the uniform change of the water film force between the string grille wire, $\frac{\partial p}{\partial z}=\frac{\Delta p}{h^{1}}, h^{l}$ is the height of the water film when it does not reach equilibrium (mm). Integrating Eq. (14) with boundary conditions of $r_{0}=r, v=0(\mathrm{~m} / \mathrm{s})$, the distribution of water mist velocity on the cross section of string grille wire is as follows:

$\frac{\mathrm{d} v}{\mathrm{~d} r_{0}}=\frac{r_{0}}{2 \mu} \frac{\Delta p}{h^{1}}$

Based on the Hagen-Poiseuille theory and its derivation, at $r_{0}=r$

$\left.\frac{\mathrm{d} v}{\mathrm{~d} r_{0}}\right|_{r_{0}=r}=\frac{r}{2 \mu} \cdot \frac{8 \mu \bar{v}}{r^{2}}=\frac{4 \bar{v}}{r}$

The internal friction force on the surface of the string grille 
wire can be obtained by replacing Eq. (16) with the Newtonian viscous fluid constitutive Eq. (13):

$\tau=\mu \frac{4 \bar{v}}{r}$

The viscous force of the adjacent string grille wire is

$F_{v}=2 \pi r h \frac{4 \mu \bar{v}}{r}=8 \pi \mu \bar{v} h$

The gravity of the water mist between the string grille wires can be expressed as

$F_{\mathrm{g}}=m g=\rho \pi r^{2} h g$

where, $h$ is the rising water film height in the string grille wire clearance $(\mathrm{mm})$ and $\rho$ is the water mist density $(\mathrm{kg} /$ $\mathrm{m}^{3}$ ). Capillary action of the water film between the string grille wires is the result of the above three forces, according to the action direction of the force, specifically

$F_{\mathrm{m}}=F_{\mathrm{v}}+F_{\mathrm{g}}$

$2 \pi r \delta \cos \theta=8 \pi \mu \bar{v} h+\rho g \pi r^{2} h$

The height of the capillary action of the water film in the gap between the string grille wires is as follows:

$h=\frac{2 r \delta \cos \theta}{8 \mu \bar{v}+\rho g r^{2}}$

From Eq. (22), the rising height of water film capillarity depends on the distance between adjacent string grille wires, the contact angle between the water film and wire, the surface tension of the water film, and the average flow velocity.

\section{Results and discussion}

\subsection{Relationship between water film area on string grille wire and dust removal efficiency}

The dust filtration efficiency of a string grille depends on the uniform filling rate of the string grille wire (Fig. 3). The filtered area of the string grille plate used is $590 \mathrm{~mm} \times 550$ $\mathrm{mm}$, the cylindrical wire diameter is $d_{\mathrm{f}}$, the wire distance is $d_{0}$, the filling rate is $\beta_{\mathrm{s}}$, and the total length of the double string grille wire diameter is

$L_{\mathrm{s}}=\frac{8 \beta_{\mathrm{s}} a b}{\pi d_{\mathrm{f}}}$

where, $L_{\mathrm{s}}$ is the total diameter and length of the double string grille wire (mm), $a$ is the height of single layer string grille wire $(\mathrm{mm})$, and $b$ is the width of the single layer string grille wire $(\mathrm{mm})$.

The working parameters are as follows: dust gas flow $Q\left(\mathrm{~m}^{3} / \mathrm{s}\right)$, dust concentration $C_{0}\left(\mathrm{~kg} / \mathrm{m}^{3}\right)$ in front of the dust

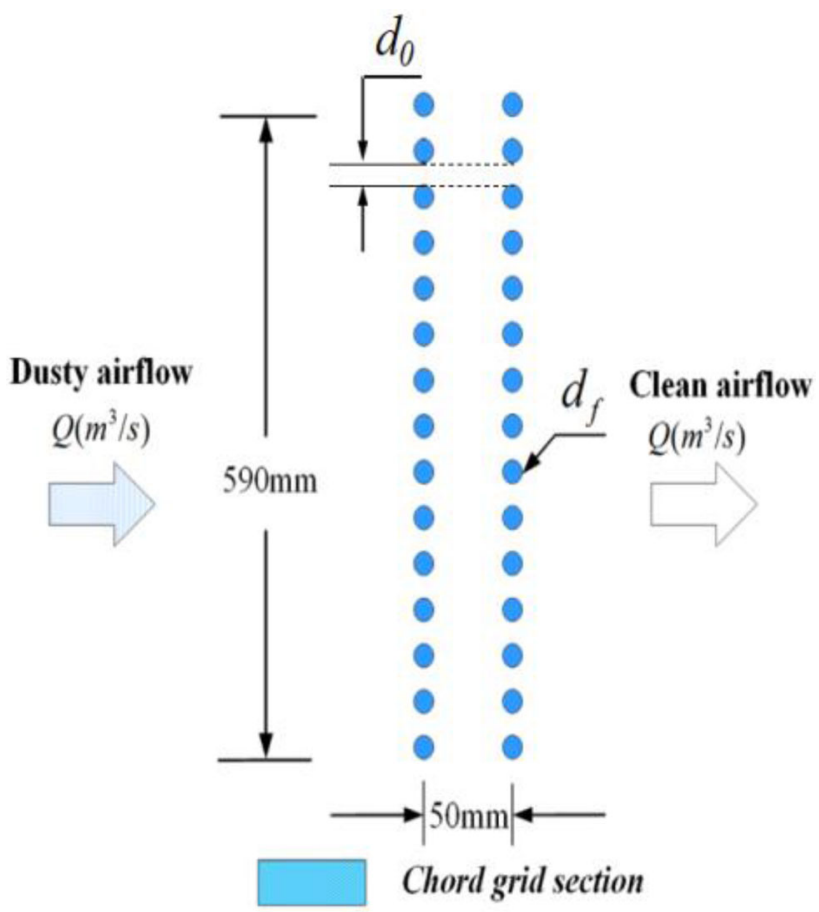

Fig. 3 Filling rate of the string grille

flow through the plate, dust concentration $C_{1}\left(\mathrm{~kg} / \mathrm{m}^{3}\right)$ after dust flow through the plate, the dimensionless filtering efficiency $\eta_{1}$, the incoming flow velocity of the filtered gas is the velocity $v(\mathrm{~m} / \mathrm{s})$ of the wind flow at an infinite distance from the string grille wire, and the velocity $\bar{v}(\mathrm{~m} / \mathrm{s})$ at the string grille wire is shown in Eq. (24). The amount of dust collected by the string grille wire per unit length $\varphi(\mathrm{g})$ is shown in Eq. (25).

$\bar{v}=\frac{v}{1-\beta_{\mathrm{s}}}=\frac{Q}{a b\left(1-\beta_{\mathrm{s}}\right)}$

$\varphi=C_{0} \eta_{1} v d_{\mathrm{f}}$

According to reference Qiang et al. (2015), considering the influence of each string grille wire dust catcher on the adjacent wire dust catcher, when the filling rate of the string grille wire is less than $75 \%$, the filtration efficiency of each string grille wire can be expressed as

$$
\begin{aligned}
\eta_{1}= & {\left[R+(0.25+0.4 R)\left(S_{\mathrm{tk}}+2 R\right)-0.0263\left(S_{\mathrm{tk}}+2 R\right)^{2}\right] } \\
& \left(0.16+10.9 \beta_{\mathrm{s}}-7 \beta_{\mathrm{s}}^{2}\right)
\end{aligned}
$$

where, $R$ is the dimensionless low interception settlement, as shown in Eq. (27) and $S_{\mathrm{tk}}$ is the dimensionless Stokes number, as shown in Eq. (28). 
$R=\frac{d_{\mathrm{p}}}{d_{\mathrm{f}}}$

$S_{\mathrm{tk}}=\frac{\rho_{\mathrm{p}} d_{\mathrm{p}}^{2} v C_{\mathrm{u}}}{18 \mu_{\mathrm{g}} d_{\mathrm{f}}}$

where, $d_{\mathrm{p}}$ is the dust particle size $(\mathrm{mm}), \rho_{\mathrm{p}}$ is the true density of dust particles $\left(\mathrm{kg} / \mathrm{m}^{3}\right), C_{\mathrm{u}}$ is the Cunningham correction coefficient, often taken as 1 , and $\mu_{\mathrm{g}}$ is the viscosity of air (Pa S).

For a string grille wire dust catcher that is uniformly distributed in the string grille, a unit filter $\mathrm{d} x$ is assumed on the string grille wire surface, and the dust concentration entering the unit is $C(\%)$. In a unit time, through the string grille with a filter area of $a \times b\left(A, \mathrm{~m}^{2}\right)$, the layer thickness can be regarded as water film thickness $L(\mathrm{~mm})$ and the total number of dust particles in the filter unit is $\beta_{\mathrm{s}} d_{\mathrm{f}} L_{\mathrm{s}} \mathrm{d} x / \frac{\pi}{4} d_{\mathrm{f}}^{2} L_{\mathrm{s}}$. Therefore, the amount of dust particles captured by the filter unit per unit time is

$-Q \mathrm{~d} C=\beta_{\mathrm{s}} \frac{d_{\mathrm{f}} L_{\mathrm{s}} \mathrm{d} x}{\frac{\pi}{4} d_{\mathrm{f}}^{2} L_{\mathrm{s}}} C A \bar{v} \eta_{1}$

When Eq. (28) is substituted into Eq. (29), the following simplification and integral can be derived:

$-\int_{C_{0}}^{C_{1}} \frac{\mathrm{d} C}{C}=\int_{0}^{L} \frac{4 \beta_{\mathrm{s}} \eta_{1}}{\pi\left(1-\beta_{\mathrm{s}}\right) d_{\mathrm{f}}} \mathrm{d} x$

The dust capture efficiency of the simplified string grille wire is

$\eta_{\mathrm{f}}=1-\exp \left[-\frac{4 \beta_{\mathrm{s}} L}{\pi\left(1-\beta_{\mathrm{s}}\right) d_{\mathrm{f}}} \eta_{1}\right]$

For a string grille wire under spray wetting conditions, the trapping of dust particles by the string grille wire and water film occurring between the strings is included in the process of dust particle capture. According to the foregoing analysis, the water film initially forms under the action of capillary condensation and adsorption condensation, and spreads out by liquid film force. The water film area is due to the formation of water mist between the wires, which is related to the vortex shedding frequency, the filling rate of wet string grille wire, and the effective saturation of water mist. Assuming that the water film between the string grille wires uniformly fills the wire gap, the water film area equation can be expressed as follows:

$A_{\mathrm{w}}=\alpha\left(1+2 S_{\mathrm{r}}\right)\left(1-\beta_{\mathrm{ws}}\right) S_{\mathrm{we}} a b$

where, $\alpha$ is the correction coefficient, considering that the water film also exists on the string wire, 1.5 is taken here, $S_{\mathrm{r}}$ is the dimensionless vortex shedding frequency, and $S_{\mathrm{we}}$ is the effective saturation of the water on the plate (dimensionless).
The vortex shedding frequency can be obtained by the relationship between vortex shedding frequency and the string grille wire Reynolds number studied by Webster et al. (1992). Under turbulent conditions, the relationship between vortex shedding frequency and the string grille wire Reynolds number is as per Eq. (33):

$S_{\mathrm{r}}=0.198\left(1-\frac{19.7}{\mathrm{Re}}\right)$

The effective saturation of water on the plate refers to water flowing in the gap, accounting for the volume of the entire plate gap, and is calculated as follows:

$S_{\mathrm{we}}=\frac{S_{\mathrm{w}}-S_{\mathrm{w} 0}}{1-S_{\mathrm{w} 0}}$

where, $S_{\mathrm{w}}$ is the water saturation, which refers to the degree to which the grille gap is filled by water mist, is the waterto-void volume ratio and is related to spray pressure. Water saturation can be obtained from the experimental data concerning spray pressure where $S_{\mathrm{w} 0}$ is the irreducible saturation of water, some of the water in the gap does not flow owing to adsorption forces. This fraction of the water is called irreducible water. The irreducible water-to-wire void volume ratio is the irreducible saturation of water, which can be calculated by the static liquid holding capacity of the wire. In the process of water film dust removal, dust is considered to be captured as long as it makes contact with the water film. Therefore, the dust removal efficiency of the water film $\eta_{\mathrm{w}}$ can be expressed as follows:

$\eta_{\mathrm{w}}=\frac{A_{\mathrm{w}}}{a b}=\alpha\left(1+2 S_{\mathrm{r}}\right)\left(1-\beta_{\mathrm{ws}}\right) S_{\mathrm{we}}$

Combining Eq. (35) with Eq. (31), the dust removal efficiency of the double wet string grille is composed of wire dust removal and water film dust removal. Therefore, the dedusting efficiency of the double-layer wet string grille is calculated as follows:

$$
\begin{aligned}
\eta_{\mathrm{wf}}= & 1-\left(1-\eta_{\mathrm{f}}\right)\left(1-\eta_{\mathrm{w}}\right) \\
= & 1-\exp \left[-\frac{1.27 \beta_{\mathrm{s}} L \eta_{1}}{\left(1-\beta_{\mathrm{s}}\right) d_{\mathrm{f}}}\right] \\
& \times\left\{1-\alpha\left(1+2 S_{\mathrm{r}}\right)\left(1-\beta_{\mathrm{ws}}\right) S_{\mathrm{we}}\right\}
\end{aligned}
$$

When the dust removal wind speed is set at $3 \mathrm{~m} / \mathrm{s}$, the string grille wire diameter is $0.23 \mathrm{~mm}$, the dust particle size is $10 \mu \mathrm{m}$, and the water irreducible saturation is 0.2 , the string grille dust removal efficiency can be expressed as follows: 


$$
\begin{aligned}
\eta_{\mathrm{wf}}= & 1-\exp \left\{\frac{-0.68}{\alpha_{\mathrm{d}}}\left[\frac{1.46 \times 10^{-4}}{\ln \left(0.9425 p^{-0.467}\right)}\right]^{\frac{1}{3}}\right. \\
& \left.\left(0.16+\frac{10.9}{1+\alpha_{\mathrm{d}}}-\frac{7}{\left(1+\alpha_{\mathrm{d}}\right)^{2}}\right)\right\} \times\left(1-\frac{A_{\mathrm{w}}}{0.325}\right)
\end{aligned}
$$

\subsection{Theoretical analysis of dust removal efficiency}

Equation (37) shows that the efficiency of dust removal is directly proportional to the water film area of the string grille. As the water film area increases, so too does the efficiency of dust removal. When the dust removal speed is $3 \mathrm{~m} / \mathrm{s}$, the spray pressure is $0.01-1.1 \mathrm{MPa}$, the string grille wire diameter is $0.23 \mathrm{~mm}$, the string grille wire distance is $0.26 \mathrm{~mm}$, the dust diameter is $10 \mathrm{um}$, and the water irreducible saturation is 0.2. Then, combining Eqs. (9), (35), and (37), the relationship between water film area and dust removal efficiency of the double wet string grille is calculated, as shown in Fig. 4. Therein, when the dust removal speed, the string grille wire distance diameter ratio, the particle size of dust, and the irreducible saturation of water are fixed, the area of the water film and the efficiency of dust removal increase with increasing spray pressure. Generally, the process can be divided into three stages. In these three stages, the growth rate of the water film area is 3-5 times higher than that of the dust removal efficiency. In particular, the spray pressure is between 0.01 and 0.20 $\mathrm{MPa}$. At this stage, the water film area and the dust removal efficiency increase faster, the average growth rate of the water film area can reach $3.05 \%$, the efficiency of dust removal efficiency is $0.70 \%$, and the spray pressure is from 0.21 to $0.40 \mathrm{MPa}$. At this stage, the growth rate of the water film area and dust removal efficiency is slow: the average growth rate of the water film area is only $0.48 \%$, and the growth rate of dust removal efficiency is only $0.14 \%$.

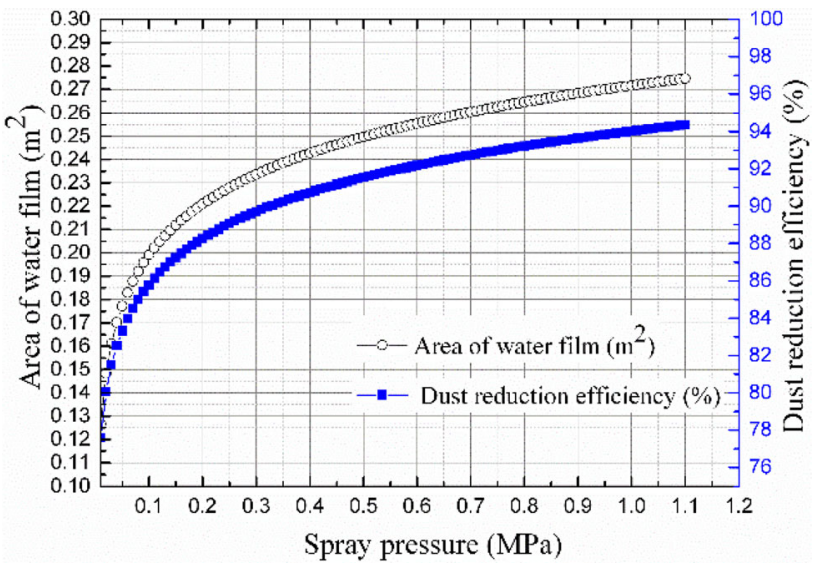

Fig. 4 Relationship between water film area and dust removal efficiency as a function of different spray pressures

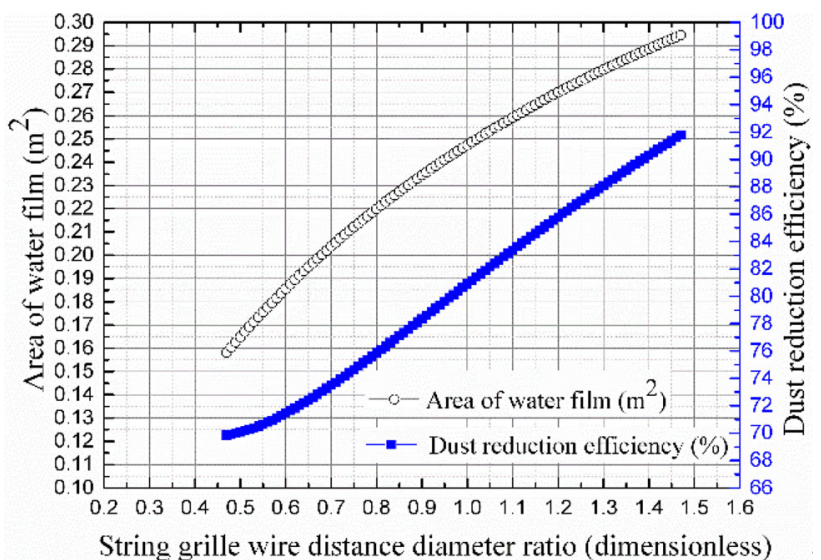

Fig. 5 Relationship between water film area and dust removal efficiency as a function of different string grille wire distance diameter ratios

When the spray pressure exceeds $0.40 \mathrm{MPa}$, the water film area and dust removal efficiency keep increasing, but the growth rate is extremely slow: the average growth rate of the water film area is only $0.17 \%$, and the growth rate of dust removal efficiency is only $0.06 \%$.

Figure 5 shows the change in the string grille wire distance diameter ratio, where the dust removal speed is $3 \mathrm{~m} / \mathrm{s}$, the spray pressure is $0.8 \mathrm{MPa}$, the string grille wire diameter is $0.23 \mathrm{~mm}$, the dust particle diameter is $10 \mathrm{um}$, the water irreducible saturation is 0.2, and Eqs. (9), (35), and (37) are combined to calculate the relationship between the water film area of the double-layer wet string grille and the dust removal efficiency. Therein, when the wind speed, spray pressure, string grille wire diameter, dust particle diameter, and water irreducible saturation are constant, the water film area and dust removal efficiency increase with increasing string grille wire distance diameter ratio, and the growth of the water film area is slightly different from that of the dust removal efficiency: as the water film area grows slower and slower, the dust removal efficiency increases first and then slows down. On the whole, the string grille wire distance diameter ratio has a non-trivial influence on the water film area and dust removal efficiency. When the ratio increases from 0.47 to 0.84 , the water film area increases from $0.158 \mathrm{~m}^{2}$ to $0.226 \mathrm{~m}^{2}$, the total growth rate is $35.798 \%$, the maximum growth rate is $1.438 \%$, and the average growth rate is $0.968 \%$; while the dust removal efficiency increases from $69.84 \%$ to $76.85 \%$, the total growth rate is $9.576 \%$, the maximum growth rate is $0.326 \%$, and the average growth rate is $0.259 \%$. At this time, the total growth rate of the water film area is 3.74times the total growth rate of dust removal efficiency. When the ratio increases from 0.85 to 1.47 , the area of the water film increases from 0.227 to $0.294 \mathrm{~m}^{2}$, the total growth rate is $26.575 \%$, the maximum growth rate is 
$0.644 \%$, and the average growth rate is $0.422 \%$; when the dust removal efficiency increases from $77.10 \%$ to $91.79 \%$, the total growth rate is $17.790 \%$, the maximum growth rate is $0.325 \%$, and the average growth rate is $0.282 \%$. At this time, the total growth rate of the water film area is 1.50 times that of dust removal efficiency.

Based on the theoretical Eq. (37) for calculating the dust removal efficiency of the wet string grille, the dust removal speed, dust particle diameter, water irreducible saturation, and string grille wire diameter are defined. The spray pressure and the string grille wire distance diameter ratio are taken as variables. Through the comparison, it is found that the effect of spray pressure on the water film area and dust removal efficiency is slightly larger than that of the string grille wire distance diameter ratio. To obtain an ideal dust removal effect, spray pressure is the key. As the spray pressure increases, the water film area also increases. The proportion of the water film in the string grille wire gap is bigger and bigger. If the dust is not captured by the string grille wire, the probability of being captured by the water film is greatly increased, thus the dust removal efficiency is greatly improved.

\section{Conclusions}

(1) The adjacent string grille wire is subjected to adhesion forces between the water mist and wire surface, resulting in the formation of a water film. Based on the Bond number, the water film formation conditions include wire spacing of $<0.34 \mathrm{~mm}$. According to mechanical equilibrium conditions, the film thickness can be derived from the Kelvin equation. Combined with the Navier-Stokes equation and the Hagen-Poiseuille law, an expression of the height of the capillary action of the water film in the string grille wire gap is constructed.

(2) Assuming that the water film between string grille wires fills the gap evenly, the effective area function of the water film is expressed by the effective saturation of the water mist, and the dedusting efficiency of the double-layer wet string grille wire is calculated.

(3) From the theoretical expression of wet string grille dust removal efficiency, we conclude that the dedusting efficiency is proportional to the water film area. A larger water film area is associated with a higher dedusting efficiency. When other factors are held constant, spray pressure and, to a lesser extent, string grille wire distance diameter are shown to affect the water film area and dust removal efficiency.
Acknowledgements We thank Esther Posner, PhD, from Edanz Group China (www.liwenbianji.cn/ac) for English language editing on an earlier draft of this manuscript. This work was supported by the 2017 Hunan Provincial Graduate Research Innovation Project of China (No. CX2017B649), the National Natural Science Foundation of China (No. 51774134), the Excellent Youth Project of Hunan Provincial Department of Education (No. 19B223), the Hunan Provincial Natural Science Foundation of China (No. 2019JJ60044), and the Hunan Provincial Natural Science Foundation of China (No. 2018JJ64028).

Open Access This article is licensed under a Creative Commons Attribution 4.0 International License, which permits use, sharing, adaptation, distribution and reproduction in any medium or format, as long as you give appropriate credit to the original author(s) and the source, provide a link to the Creative Commons licence, and indicate if changes were made. The images or other third party material in this article are included in the article's Creative Commons licence, unless indicated otherwise in a credit line to the material. If material is not included in the article's Creative Commons licence and your intended use is not permitted by statutory regulation or exceeds the permitted use, you will need to obtain permission directly from the copyright holder. To view a copy of this licence, visit http://creativecommons. org/licenses/by/4.0/.

\section{References}

Adamson AW, Gast AP (1997) Physical chemistry of surfaces, 6th edn. Wiley Interscience Press, New York

Agranovski IE, Braddock RD (1998) Filtration of liquid aerosols on wettable fibrous filters. AICHE J 44(12):2775-2783

Chen XJ, Yao GQ (2017) An improved model for permeability estimation in low permeable porous media based on fractal geometry and modified Hagen-Poiseuille flow. Fuel 210(12):748-757

Fairs LG (1958) High efficiency fibre filters for the treatment of fine mists. Trans Inst Chem Eng 36:476-485

Gao SQ, Liu HP (2010) Capillary mechanics. Science Press, Beijing

Gong LH (2002) Theoretical and experimental study on dust removal technology of wet vibrating fiber grille. Northeast University, Shenyang

Han H, Wang PF, Liu RH et al (2020) Experimental study on atomization characteristics and dust-reduction performance of four common types of pressure nozzles in underground coal mines. Int J Coal Sci Technol 7(3):581-596

Jakub MG (2015) A simple numerical model of pressure drop dynamics during the filtration of liquid aerosols on fibrous filters. Sep Sci Technol 50(13):2015-2022

Jakub MG, Jackiewicz A, Werner L, Jakubiak S (2016) Consecutive filtration of solid particles and droplets in fibrous filters. Sep Purif Technol 170(10):234-240

Jin LZ, Li JP, Su YF et al (2010) Theory of mine dust prevention and control. Science Press, Beijing

Li YC, Zhang YH, Xiong SS (2011) Study on dedusting technology with high-pressure atomizing and vibrating fiber grille. Nonferrous Met (min Sect) 63(1):58-61

Li JL, Zhou FB, Li SH (2017) Experimental study on the dust filtration performance with participation of water mist. Process Saf Environ Prot 109:357-364

Lin N, Li GL, Wang HD et al (2015) Research progress of surface free energy's computing methods and the influence on the properties of material surface. Mater Rev 29(11):30-35 
Probmtein RF, Dai GC, Fang GN et al (1992) Introduction to physicochemical fluid dynamic. East China Institute of Chemical Technology Press, Shanghai

Qiang N, Ji XL, Xu B et al (2015) Air pollution control engineering. Chemical Industry Press, Beijing

Reed WR, Joy GJ, Shahan M, Klima S, Ross G (2019) Laboratory results of a 3rd generation roof bolter canopy air curtain for respirable coal mine dust control. Int $\mathrm{J}$ Coal Sci Technol 6(1):15-26

Ryan MH, King AJC, Kasper G et al (2013) Computational fluid dynamics (CFD) simulation of liquid aerosol coalescing filters. J Aerosol Sci 61(11):36-49

Tan C, Jiang ZA (2015) Experimental study on dust-control by water curtain in underground mines. J China Univ Min Technol 44(6):1002-1007

Tanaka K, Iwamoto K (2016) Effective shear displacement on lateral adhesion force of a liquid bridge between separated plates. Tribol Lett 64(1):1-7

Wang SL (2007) Physical chemistry, 3rd edn. Metallurgical Industry Press, Beijing

Wang PF, Shi YJ, Zhang L et al (2019) Effect of structural parameters on atomization characteristics and dust reduction performance of internal-mixing air-assisted atomizer nozzle. Process Saf Environ Prot 128:316-328

Webster S, McBride R, Barton J et al (1992) Air flow measurement by vortex shedding from multimode and monomode optical fibers. Meas Sci Technol 13(2):210-216
Wu CF, Li YJ, Chen ZY et al (2017) Experimental study on influencing factors of dust removal performance for dust collector with charged water mist vibration fiber grille. J Saf Sci Technol 13(3):162-168

Yao HF, Wang HY, Li YC et al (2020) Three-dimensional spatial and temporal distributions of dust in roadway tunneling. Int $\mathrm{J}$ Coal Sci Technol 7(1):88-96

Zhang XX, Zhang ZT, Zhang WF et al (2018) Comparison of five eddy viscosity turbulence models in numerical simulation of flow over a two-dimensional square cylinder. Acta Aerosol Sin 36(2):339-349

Zhang T, Deji J, Shaocheng Ge, Jiren W, Xiangxi M, Shuaishuai R (2020) Numerical simulation of the dimensional transformation of atomization in a supersonic aerodynamic atomization dustremoving nozzle based on transonic speed compressible flow. Int J Coal Sci Technol 7(3):597-610

Zhou G, Zhang Q, Bai RN et al (2017) The diffusion behavior law of respirable dust at fully mechanized caving face in coal mine: CFD numerical simulation and engineering application. Process Saf Environ Prot 106:117-128

Zhou G, Zhang Q, Hu Y et al (2020) Dust removal effect of negatively-pressured spraying collector for advancing support in fully mechanized coal mining face: numerical simulation and engineering application. Tunnell Undergr Space Technol 95 art. no. 103149

Zhuang DW, Yang YF, Hu HT et al (2016) Visualization and prediction model on shape of liquid bridge. CIESC J 67(6):2224-2229 